\title{
Discussion on the Problems of Leakage Fractal Model of Mechanical Seal
}

\author{
Long Wei ${ }^{1 * *}$, Wenrui Teng ${ }^{2}$, Penggao Zhang ${ }^{1}$, Guifang Fang ${ }^{1}$ and Xingzhong Chang ${ }^{2}$ \\ ${ }^{1}$ Fluid Sealing Measurement and Control Engineering Research and Development Center in Jiangsu Province, Nanjing Polytechnic \\ Institute, Nanjing 210048, China \\ ${ }^{2}$ Henan Vocational College of Chemical Technology, Zhengzhou 450042, China
}

\begin{abstract}
Leakage rate is a key parameter for evaluating the mechanical seal performance. The leakage process of mechanical seal is very complicated. Influencing factors of contact mechanical seal are many, and the leakage is a variable associated with operating time rather than the steady parameter. Therefore, establishing the leakage model of mechanical seal is difficult. In this paper, surface topography fractal charactering method and the expression of fractal parameter calculated by the structure function of mechanical seal are given. The leakage fractal model is listed and its 6 problems are discussed.
\end{abstract}

\section{Introduction}

Leakage rate is a key parameter for evaluating the mechanical seal performance. Mechanical seal leakage is caused by lots of reasons, and mainly through the radial clearance between friction pair in normal operation. At present, there are two types leakage models of contact mechanical seal, one type is empirical formula and numerical calculation model based on surface topography charactering parameters [1-3], and the other is leakage model based on fractal geometry theory[4-5]. Leakage rate empirical formula is simple, easy to use, and can visually reflects the relationship between the leakage rate and the main influencing factors, however, it can only be used for estimating the leakage rate because of big error. Numerical calculation model can comprehensively reflect the influences of the geometric parameters, the surface topography parameters and working parameters on the leakage rate, but it needs to be iterated by computer programming. Furthermore, the leakage rate calculated by numerical calculation model is generally more bigger[6].

\section{Fractal characteristics of surface topology of mechanical seal}

Surface topography has a great influence on leakage rate of mechanical seal[6]. Studies simplified asperity on the end face of friction pair of mechanical seal as hemispheroid of which peak height follows Gaussian distribution, and characterized surface topography which is regarded as a stable random process by statistical parameters of arithmetic average deviation of profile, root-mean-square deviation of profile, etc[1-3]. Because the simplified model of surface topography is too simple, and statistical parameters are obtained under certain measurement conditions and affected greatly by sensitivity of the measuring instruments and sampling length, so it can't reflects the complexity of real surfaces, non-stable random characteristic, and multiply scaling feature of the roughness, etc. It is necessary to look for a scale-independent parameter instead of scale-dependent statistical parameters for charactering rough surface topography.

Studies[7-12] have shown that profile curves of the end faces of mechanical seal before and after wear have isotropic self-affine fractal property with no relevance to scale, and it can be characterized and simulated by the continuous and non-differentiable WeierstrassMandelbrot ( W-M ) function with self-affine fractal property. The expression was[13]:

$$
z(x)=G^{D-1} \sum_{n=n_{l}}^{\infty} \frac{\cos \left(2 \pi \gamma^{n} x\right)}{\gamma^{(2-D) n}}
$$

Where $z(x)$ is the deviation of the surface profile from its mean line, $x$ is the lateral distance, $G$ is a characteristic length scale of the surface, $D$ is the fractal dimension of the surface profile (for a physically continuous surface $1<D<2), \gamma^{n}$ are the discrete frequency $\operatorname{modes}(\gamma>1), n_{l}$ corresponds to the low cut-off frequency of the profile and is given by $\gamma^{n_{l}}=1 / L$, and $L$ is the sampling length. Choosing $\gamma=1.5$ can provide both phase randomization and high spectral density.

There are many methods for calculating the fractal parameters, and the results obtained by structure function method is more accurate for larger fractal dimension[13]. Fractal parameters of surface profile of mechanical seal should be calculated by structure function because seal rings are manufactured by grinding, and the fractal dimension is larger.

$D$ and $G$ can be determined by structure function $S(\tau)$. Analog signal of profile height $z(x)$ measured by profiler was put into computer after A/D conversion. Sampling interval $\Delta t$ of computer was given. $N$ points were acquired on sampling length $l r$. There was $z\left(x_{i}\right)=Z_{i}(i=0,1$, $2,3, \cdots, N-1)$. Assuming $\tau=n \Delta t(n=0,1,2,3, \cdots)$, and the structure function was:

$$
S(\tau)=S(n \Delta t)=\frac{1}{N-n} \sum_{i=0}^{N-n}\left(z_{i+n}-z_{i}\right)^{2}
$$

A line can be obtained by plotting with $S(\tau)$ and $\tau$ in double logarithm coordinate system. Fractal parameters $D$ and $\mathrm{G}$ can be calculated by slope $k_{s}$ and intercept $B$. 


$$
\begin{gathered}
D=2-\frac{k_{s}}{2} \\
G=10^{\frac{B}{2(D-1)}}\left\{\frac{(4-2 D) \ln \gamma}{\Gamma(2 D-3) \sin [(2 D-3) \pi / 2]}\right\}^{\frac{1}{2(D-1)}}
\end{gathered}
$$

\section{Proposition of leakage fractal model of mechanical seal}

Sun, et al. introduced the fractal theory into the study of contact mechanical seal firstly. The contact between hard and soft ring was simplified as the contact between the rigid ideal smooth surface and the rough surface (see Fig. 1). Regarding cavities in the annular section at the outer diameter of the end face as micro-leakage channel section, charactering it by fractal parameters, and the mechanical seal leakage model related to the operating time was established based on the changing of surface topography[4-5].

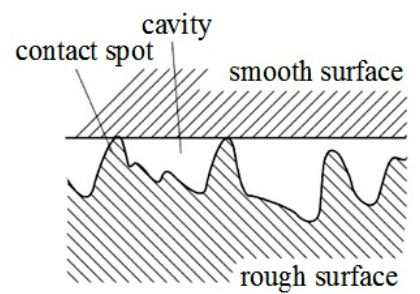

Figure 1. Contact model of end face of mechanical seal

Sun, et al. assumed that the fluid flowing through contact interface in the clearance between the end faces of mechanical seal was incompressible, viscous and steady laminar flow between the leakage channels (see Fig. 2). According to Navier-Stokes equation, the leakage rare, $q$, of single leakage channel can be calculated under the conditions of the changes of viscosity, the rotation of fluid and the changes of seal ring curvature were neglected, and $q$ was[4]

$$
q=\frac{p_{2}-p_{1}}{9 \pi \eta\left(r_{2}-r_{1}\right)} G^{3[D(t)-1]} a_{s}^{[7-3 D(t)] / 2}
$$

Figure 2. Leakage channel model

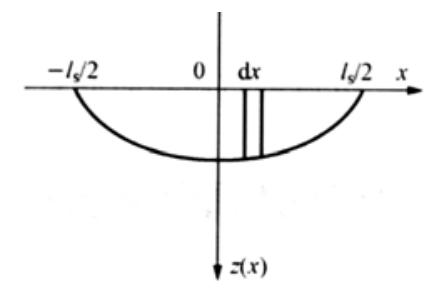

Where $p_{1}$ and $p_{2}$ are inside and outboard medium pressures of seal face respectively, $r_{1}$ and $r_{2}$ are inner and outer radii of seal face respectively, $\eta$ is the dynamic viscosity of fluid flowing through contact interface, $D$ is the fractal dimension of the end face profile of the soft ring, $G$ is a characteristic length scale of the end face profile of the soft ring, $a_{s}$ is a micro cavity area in contact face, and $t$ is the operating time of mechanical seals.
The contact cavity area distribution function $n\left(a_{s}\right)$ between the surfaces and the sum of micro cavity areas $A_{s}$ between contact faces were[4]:

$$
\begin{gathered}
n\left(a_{s}\right)= \begin{cases}\frac{D}{2} \psi^{(2-D) / 2} a_{s l}^{D / 2} a_{s}^{-(D+2) / 2}, & 0<a_{s}<a_{s l} \\
0, & a_{s l}<a_{s}<+\infty\end{cases} \\
A_{s}=A_{a}-A_{r}=\frac{D}{2-D} \psi^{(2-D) / 2} a_{s l}
\end{gathered}
$$

Where $\psi$ is the domain extension factor for microcontact size distribution, $a_{s l}$ is the area of the largest micro cavity, $A_{s}$ is the sum of the micro cavity areas between contact faces, $A_{a}$ is the apparent contact area, and $A_{r}$ is the true contact area.

The total volume leakage rate $Q$ can be obtained by Eqs.(5)-(7) [5]

$$
\begin{aligned}
Q & =\int_{0}^{a_{s l}} q n\left(a_{s}\right) \mathrm{d} a_{s} \\
& =\frac{\left(p_{2}-p_{1}\right) D(t) G^{3[D(t)-1]}}{9 \pi \eta\left(r_{2}-r_{1}\right)[7-4 D(t)]} \psi^{\frac{2-D(t)}{2}} a_{s l}^{[7-3 D(t)] / 2}
\end{aligned}
$$

The maximum micro cavity area $a_{s l}$ can be obtained by the relationship between Eq.(7) and total load in the contact surfaces, and real contact area.

The relationships between the loads and contact areas of elastic, elastic-plastic and plastic contact spots in the friction pair of mechanical seal were[5]

$$
\begin{gathered}
F_{e}(a)=\frac{4 \sqrt{\pi}}{3} E G^{(D-1)} a^{(3-D) / 2} \\
F_{e p}(a)=\frac{2 a}{3} E \varphi_{0}\left[2+\ln \left(\frac{\sqrt{\pi} G^{(D-1)} a^{(1-D) / 2}}{3 \varphi_{0}}\right)\right] \\
F_{p}(a)=E K \varphi_{0} a
\end{gathered}
$$

Where $F_{e}(a)$ is the load on the elastic contact spot, $F_{e p}(a)$ is the load on the elastic-plastic contact spot, $F_{p}(a)$ is the load on the plastic contact spot, $a$ is the area of the micro contact spot; $E$ is the comprehensive elastic modulus, $\varphi_{0}$ is the material property constant, and $K$ is a ratio of the hardness $H$ to the yield strength $\sigma_{y}$ of the soft material.

The critical contact area $a_{p e}$ of plastic deformation transforming to elastic-plastic deformation was[5]

$$
a_{p e}=G^{2}\left(\frac{\sqrt{\pi}}{30 \varphi_{0}}\right)^{2 /(D-1)}
$$

The critical contact area $a_{c}$ of elastic-plastic deformation transforming to elastic deformation was[5]

$$
a_{c}=G^{2}\left(\frac{4}{3 \sqrt{\pi} K \varphi_{0}}\right)^{2 /(D-1)}
$$


Therefore, the relationship between total load and real contact area in the whole contact surfaces is[5]

$$
\begin{gathered}
F_{g}=\int_{a_{c}}^{a_{l}} F_{e}(a) n(a) \mathrm{d} a+\int_{a_{p e}}^{a_{c}} F_{e p}(a) n(a) \mathrm{d} a+ \\
\int_{0}^{a_{p e}} F_{p}(a) n(a) \mathrm{d} a
\end{gathered}
$$

Where $a_{l}$ is the area of the largest contact spot, $a_{l}=\frac{2-D}{D} \psi^{(D-2) / 2} a_{r}$. where $n(a)$ is the size-distribution of contact spots area.

\section{Problems of leakage fractal model of the end faces of mechanical seal}

Because mechanical seal mechanism is so complicated, and study of mechanical seal by fractal theory does not go further enough, so the leakage fractal model also has the following main problems.

4.1. The influences of fluid film pressure between the end faces of mechanical seal were neglected

It can be seen from Eq.(14) that the total load (closing force) $F_{\mathrm{g}}$ is supported by reaction force of asperities. The research results of contact mechanical seal have indicated that when the end faces were in the boundary friction, the measured pressure of liquid film between the end faces was close to zero, and the load was almost all supported by asperities[1]. When the end faces were in the mixed friction, the load was supported by liquid film and contact asperities[1-3]. Therefore, the leakage fractal model is suitable for mechanical seal is in boundary friction, but there is a large error for mechanical seal is in mixed friction

4.2 The influences of friction between the contact asperities of mechanical seal were neglected

The relationship between load and real contact area between the end faces of mechanical seal neglected the influences of friction between the contact asperities in [45]. However, study has indicated that the friction between the contact asperities had a great influence on the real contact area when the surfaces were smoother[14].

\subsection{There is an abrupt change of load at the critical point of deformation transformation of the asperities in mechanical seal}

Substituting Eq.(12) into Eqs.(10) and (11) respectively, the ratio of elastic-plastic load to plastic load can be obtained at the critical point of plastic deformation transforming to elastic-plastic deformation, and $\frac{F_{e p}\left(a_{p e}\right)}{F_{p}\left(a_{p e}\right)}=\frac{2(2+\ln 10)}{3 K}$. Substituting Eq.(13) into Eqs.(9) and (10) respectively, the ratio of elastic load to elasticplastic load can be obtained at the critical point of elasticplastic deformation transforming to elastic deformation, and $\frac{F_{e}\left(a_{c}\right)}{F_{e p}\left(a_{c}\right)}=\frac{3 \pi K}{2[2+\ln (\pi K / 4)]}$. If $H=30 \mathrm{MPa} 、 \sigma_{y}=50$ $\mathrm{MPa}[5]$, and $K=0.6$. Hence, there are $F_{e p}\left(a_{p e}\right) / F_{p}\left(a_{p e}\right)=4.78$ at the critical point of plastic deformation transforming to elastic-plastic deformation and $F_{e}\left(a_{c}\right) / F_{e p}\left(a_{c}\right)=2.27$ at the critical point of elasticplastic deformation transforming to elastic deformation. There is an abrupt change of contact load.

4.4 Micro cavity area distribution function, $n\left(a_{s}\right)$, between the end faces was used to replace the micro leakage channel section area distribution function

The leakage fractal model regarded cavities on the annular section at the outer diameter of the end face as micro leakage channel section, and Eq.(5) was the leakage rare fractal expression of single leakage channel. However, micro cavity area distribution function, $n\left(a_{\mathrm{s}}\right)$, between the end faces in Eq.(6) was used to replace the micro leakage channel section area distribution function when the total volume leakage rate $Q$ was calculated by $\int_{0}^{a_{s l}} q n\left(a_{s}\right) \mathrm{d} a_{s}$ in Eq. (8).

\subsection{The influences of the tortuosity of leakage path were neglected}

There are contacts between the asperities of the end faces and sliding between two end faces, and the radial leakage path is a tortuous channel (see Fig. 3) rather than a straight way for contact mechanical seal. However, when leakage rate expression, namely Eq.5, of single leakage channel was deduced in [4-5], the leakage path was simplified as a radial straight way, and the influences of the tortuosity of leakage path were neglected.

\subsection{The influences of the temperature of the end face were neglected}

It can be known from Eq.(8) that the fluid viscosity $\eta$ has a direct influence on leakage rate. Viscosity changes with the changes of temperature of the end face of mechanical seal[15]. However, the influences of the temperature of the end face were neglected in [4-5].

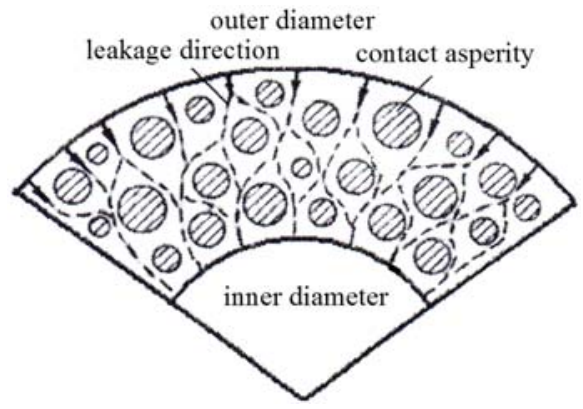

Figure 3. Leakage path 


\section{Conclusions}

The leakage process of mechanical seal is very complicated. Influencing factors of contact mechanical seal are many, and the leakage is a variable associated with operating time rather than the steady parameter. Therefore, establishing the leakage model of mechanical seal is difficult.

The proposition of contact mechanical seal leakage model based on fractal theory provides a new means for studying mechanical seal, reveals the relationships between the leakage rate and sealant pressure, sealant viscosity, unit load, property of material of seal ring and surface topography. The results indicated that leakage rate is an unsteady parameter, and provided a theoretical foundation for proposing the measures for reducing leakage and engineering practice. Because the study of mechanical seal based on fractal theory does not go further enough, so the leakage fractal model has some problems. Further theoretical study and experimental study must be needed for establishing a correct leakage model of contact mechanical seal.

\section{Acknowledgement}

This research was financially supported by the Brand Specialty Construction Project of the Jiangsu Higher Education Institutions of China (Grant No. PPZY2015C232) and Key Scientific Research Project of Henan Higher Education Institutions of China (Grant No. 15B530001).

\section{References}

[1] J.D. Summers-Smith, Mechanical seal practice for improved performance, Mechanical Engineering Publications for the Institution of Mechanical Engineers, London, 1992.

[2] I. Etsion, I. Front, A model for static sealing performance of end face seals, Tribology Transactions. 37 (1994) 111-119.

[3] L.Q. Wang, X.K. Meng, W.P. Dai, Analysis on sealing performance and fluid-solid coupling model of contacting mechanical seals, Journal of Engineering Thermophysics. 29 (2008) 1864-1866.

[4] J.J. Sun, B.Q. Gu, L. Wei, Leakage model of contacting mechanical seal based on fractal geometry theory, Journal of Chemical Industry and Engineering. 57 (2006) 1626-1631.

[5] J.J. Sun, L. Wei, X. Feng, Leakage prediction method for contacting mechanical seals with parallel faces, Chinese Journal of Mechanical Engineering. 23 (2010) 7-15.

[6] L. Wei, X.Z. Chang, P.G. Zhang, Progress of study on leakage models of contacting mechanical seal end faces, Fluid Machinery. 40 (2012) 36-40.

[7] L. Wei, B.Q. Gu, X. Feng, Contact fractal model for friction faces of mechanical seals, Chinese Journal of Mechanical Engineering. 60 (2009) 2543-2548.
[8] L. Wei, B.Q. Gu, J.J. Sun, Optimization of surface fractal dimension of friction pair in mechanical seals, China Surface Engineering. 61 (2010) 132-136.

[9] L. Wei, X.Z. Chang, P.G. Zhang, Fractal characterization of changing of surface topography of the end face of soft ring in running-in procedure for mechanical seal, China Surface Engineering. 24 (2011) 78-82.

[10]L. Wei, B.Q. Gu, P.G. Zhang, Contact characterizations of end faces in mechanical seals running-in, CIESC Journal. 63 (2012) 3202-3207.

[11]L. Wei, B.Q. Gu, Q.H. Liu, Correction of contact fractal model for friction faces of mechanical seals, CIESC Journal. 64 (2013) 1723-1729.

[12]L. Wei, B.Q. Gu, P.G. Zhang, Average film thickness prediction of end faces in contacting mechanical seals in running-in period, CIESC Journal. 64 (2013) 41374142.

[13]A. Majumdar, B. Bhushan, Role of fractal geometry in roughness characterization and contact mechanics of surfaces, ASME Journal of Tribology. 112 (1990) 205-216.

[14]L. Wei, Q.H. Liu, P.G. Zhang, Sliding friction surface contact mechanics model based on fractal theory, Journal of Mechanical Engineering. 48 (2012) 106-113.

[15]L. Wei, B.Q. Gu, P.G. Zhang, Average temperature coupling calculation method for end faces of contact mechanical seals, CIESC Journal. 65 (2014) 35683575 . 